\title{
Myeloperoxidase-rich Ly-6C+ myeloid cells infiltrate allografts and contribute to an imaging signature of organ rejection in mice
}

Filip K. Swirski, Moritz Wildgruber, Takuya Ueno, Jose-Luiz Figueiredo, Peter Panizzi, Yoshiko Iwamoto, Elizabeth Zhang, James R. Stone, Elisenda Rodriguez, John W. Chen, Mikael J. Pittet, Ralph Weissleder, and Matthias Nahrendorf

Center for Systems Biology, Massachusetts General Hospital and Harvard Medical School, Boston, Massachusetts, USA.

\begin{abstract}
Rates of graft rejection are high among recipients of heart transplants. The onset and progression of clinically significant heart transplant rejection are currently monitored by serial biopsy, but this approach is highly invasive and lacks sensitivity. Here, we have developed what we believe to be a new technique to measure organ rejection noninvasively that involves the exploration of tissue-infiltrating leukocytes as biomarker sources for diagnostic imaging. Specifically, we profiled the myeloid response in a murine model of heart transplantation with the aim of defining and validating an imaging signature of graft rejection. $\mathrm{Ly}-6 \mathrm{C}$ hi monocytes, which promote inflammation, accumulated progressively in allografts but only transiently in isografts. Ly- $6 \mathrm{C}^{\text {lo }}$ monocytes, which help resolve inflammation, did not accumulate, although they composed the majority of the few remaining monocytes in isografts. The persistence of $\mathrm{Ly}-6 \mathrm{C}^{\mathrm{hi}}$ monocytes in allografts prompted us to screen for a Ly-6 Chi monocyte-associated imaging marker. Low-density array data revealed that Ly- $6 \mathrm{C}^{\text {hi }}$ monocytes express 10-fold higher levels of myeloperoxidase (MPO) than Ly-6 $\mathrm{C}^{\mathrm{lo}}$ monocytes. Noninvasive magnetic resonance imaging of MPO with an MPO-activatable Gd-chelate revealed a spatially defined T1-weighted signal in rejected allografts but not in isografts or MPO-deficient allograft recipients. Flow cytometry, enzymography, and histology validated the approach by mapping MPO activity to $\mathrm{Ly}-6 \mathrm{C}$ hi monocytes and neutrophils. Thus, MPO imaging represents a potential alternative to the current invasive clinical standard by which transplants are monitored.
\end{abstract}

\section{Introduction}

The ability to monitor noninvasively the host response to a graft is an unmet goal in heart transplantation and essential to titrate immunosuppressive therapy. Serial endomyocardial biopsies are the current clinical standard for graft monitoring; a patient initially undergoes 3-4 individual biopsies every second week after transplantation (1). The invasive procedure is deemed necessary because of the high rates of graft rejection (20\% in the first year) (2), but it can give rise to bleeding, infection, arrhythmia, pneumothorax, carotid puncture, ventricular perforation, and tricuspid insufficiency $(3,4)$. Moreover, it is error prone due to the limited sampling and causes discomfort and anxiety. Thus, there is a need for alternative approaches that survey transplanted organs quantitatively and noninvasively and provide spatially encoded data.

Graft rejection is characterized by an influx of multiple cell types, including cells of the myeloid lineage, among them monocytes. Monocytes are circulatory cells that give rise to tissue macrophages and some subsets of dendritic cells during inflammation and are therefore important to the overall inflammatory cascade. Studies have shown, however, that monocytes are heterogeneous in humans and mice. Mouse Ly-6C $\mathrm{C}^{\text {hi }} \mathrm{CCR} 2^{\text {hi }}$ monocytes are analogous to human $\mathrm{CD} 16^{1 \circ} \mathrm{CD} 14^{+}$monocytes and promote inflammation during chronic diseases like atherosclerosis (5-7) and in response to myocardial infarction $(8)$ and infection $(9,10)$, while so-called Ly-6 $\mathrm{C}^{\text {lo }} \mathrm{CCR} 2^{\text {lo }}$ monocytes are analogous to $\mathrm{CD} 16^{\mathrm{hi}} \mathrm{CD} 14^{\text {lo/neg }}$ and

Conflict of interest: Ralph Weissleder owns shares of VisEn Medical.

Citation for this article: J Clin Invest. 2010;120(7):2627-2634. doi:10.1172/JCI42304. participate in the resolution of inflammation (11-14). It is less well understood how subsets participate in transplantation, but their role is likely significant given that myeloid cells can be as numerous as lymphocytes in rejected allografts $(15,16)$ and have been linked to transplant function (17).

In this study, we profiled the host leukocyte response after cardiac allograft transplantation. We show that acutely rejected hearts accumulate Ly-6Chi monocytes preferentially and progressively, whereas isografts and allografts treated with immunosuppressive therapy show dominance of Ly-6C $\mathrm{C}^{\text {lo }}$ monocytes. The other major myeloid cells, neutrophils, infiltrate the heart transiently, irrespective of histocompatibility, indicating a nonspecific response to surgery. Using this information, we then mined a low-density transcriptome array of blood monocytes (18) for clinically suitable molecular imaging targets and found that Ly-6Chi but not Ly-6 $\mathrm{C}^{\text {lo }}$ monocytes express high levels of myeloperoxidase (MPO), an enzyme that is also present in neutrophils in high quantities. Thus, we hypothesized that imaging MPO activity by MRI can monitor organ rejection and discriminate rejection from tolerance induced by immunosuppressive therapy noninvasively and with higher resolution than afforded by biopsies.

\section{Results}

Ly-6C myeloid cells dominate the lenkocyte response to allograft rejection. We first profiled the myeloid cell population in allo- versus isografts (Figure 1). We used a heterotopic heart transplant model in which the recipient mouse received a fully MHC I- and II-mismatched allograft (BALB/c donor, $\mathrm{C} 57 \mathrm{BL} / 6$ recipient) or a control 
A No Tx

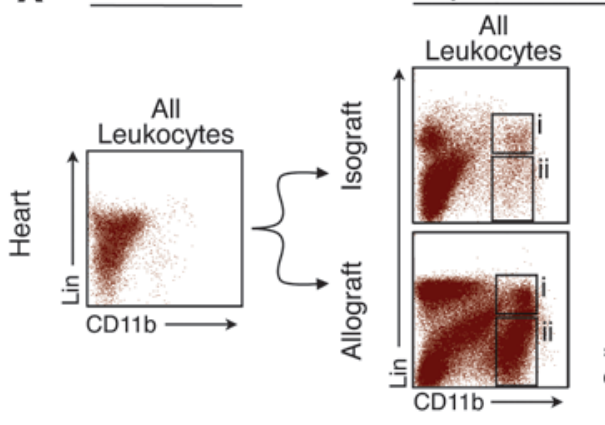

$\underline{\text { Day } 7 \text { after } T x}$

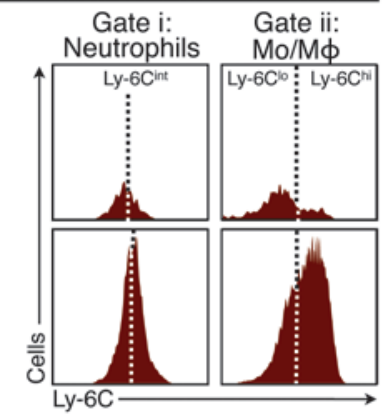

B
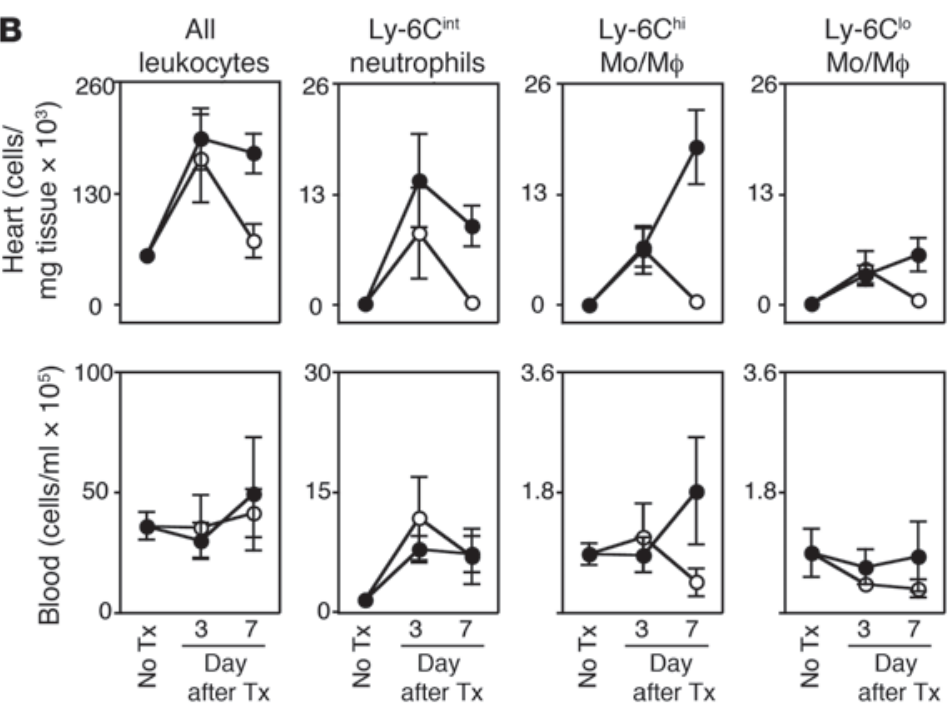

C
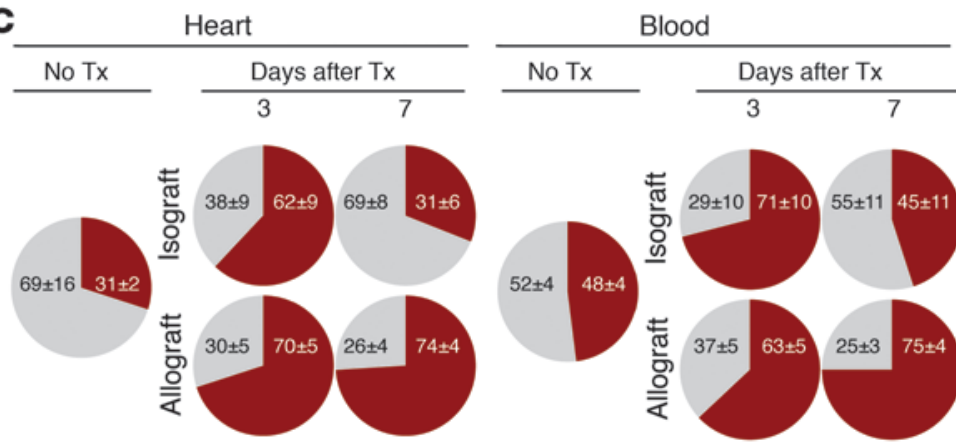

Ly-6C $C^{\text {hi }} \mathrm{Mo} / \mathrm{M} \phi$

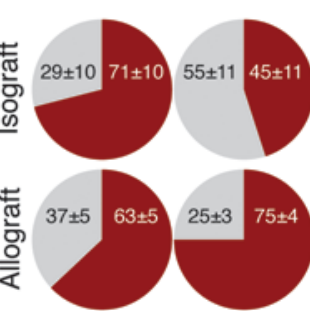

Ly-6C $\mathrm{Cl}^{\mathrm{o}} \mathrm{Mo} / \mathrm{M \phi}$

Figure 1

Myeloid cell profile in transplant rejection. (A) Flow cytometric analysis of cell suspensions retrieved from native hearts and iso- and allografted hearts 7 days after transplantation. Lin, CD90/B220/CD49b/NK1.1/Ly-6G. Histograms on right depict the level of Ly-6C expression in respective populations. A representative experiment of 5 is shown. Tx, transplantation. (B) Time course of myeloid cell presence in heart and blood of allograft (black circles) and isograft (white circles) recipients. Animals receiving no transplants served as controls. (C) Ratio of Ly- $6 C^{\text {hi }}$ vs. Ly- $6 \mathrm{C}^{10}$ monocytes in the native heart, in isografts and allografts, and in blood. Data are shown as mean \pm SEM; $n=3-5$ per group.

response depended on histocompatibility. Comparison of monocyte subsets in grafts revealed that control isografts on day 7 contained more than 2 times more Ly-6Clo monocytes than their Ly-6Chi counterparts $(69 \% / 31 \%)$, which is similar to the proportion of these cells in myocardium in the steady state (Figure 1C). However, inflammatory Ly- $6 \mathrm{C}^{\text {hi }}$ monocytes dominated the rejected allografts (74\%). The relative proportions of monocyte subsets in the transplanted organ mirrored those in peripheral blood (Figure 1C). These data indicate that Ly-6C ${ }^{\text {hi }}$ monocytes dominate the myeloid response in transplant rejection.

Ly-6C-associated MPO is a biomarker for graft rejection. Given that accumulation of Ly-6Chi monocytes was associated with rejection, we hypothesized that the cells could be targeted for diagnostic imaging. We recently profiled the transcriptome of murine monocyte subsets (18). Mining these data for potential molecular imaging targets that are expressed selectively by 1 subset of monocytes, we found that MPO, an enzyme with microbicidal and viricidal properties, was expressed 9.8 times more in Ly-6 $\mathrm{C}^{\text {hi }}$ versus Ly- $6 \mathrm{C}^{\text {lo }}$ monocytes $(P<0.01)$. To confirm that the differences at the transcriptional level extended to protein and activity levels, we evaluated MPO expression and activity in cells retrieved from rejected heart grafts. Flow cytometric (Figure 2, A and B) and enzymographic (Figure 2C) assays showed that Ly-6Chi monocytes and neutrophils, but not lymphocytes and Ly-6Clo monocytes, expressed MPO in blood and heart. By multiplying the cell number (Figure 1B) by the flow cytometric MPO staining intensity for each of the leukocyte popula-

syngeneic isograft (C57BL/6 donor, C57BL/6 recipient) via anastomoses of the donor aorta and vena cava superior to the recipient abdominal aorta and vena cava inferior, respectively. One week after transplantation, allografts accumulated massive numbers of $\mathrm{CD}_{11 \mathrm{~b}^{+}}$myeloid cells consisting of Ly- $6 \mathrm{C}^{\mathrm{hi}}$ monocytes and $\left(\mathrm{Ly}-6 \mathrm{C}^{+}\right)$ neutrophils (Figure 1A). Isografts, in contrast, accumulated few myeloid cells, many of which were Ly- $6 \mathrm{C}^{\text {lo }}$ monocytes. Time-course quantitative analysis of grafts and blood revealed a neutrophil-rich surge to the graft on day 3 that was independent of histocompatibility and subsided by day 7 , indicating a nonspecific response to ischemia reperfusion injury incurred by the transplant procedure (Figure 1B). Monocyte accumulation, however, was progressive in allografts and transient in isografts, indicating that the monocyte tions present in grafts, we predicted that Ly- $6 \mathrm{C}^{\text {hi }}$ monocytes and neutrophils account for nearly the entire MPO signal in rejected allografts (Figure 2B). Using the same calculation, isografts, in contrast, had a 27 -fold lower cellular MPO content per mg myocardium (Figure 2B). To validate that these findings corresponded to enzyme function, we flow sorted cell populations from 7-day allografts and found that only $\mathrm{Ly}-6 \mathrm{C}^{\text {hi }}$ monocytes and neutrophils exhibited MPO activity. We concluded that MPO's strong association with inflammatory Ly- $6 \mathrm{C}^{+}$myeloid cells and lack thereof with reparative Ly- $6 \mathrm{C}^{\mathrm{lo}}$ monocytes rendered it a prospective candidate for diagnostic imaging of graft rejection. Importantly, the imaging approach does not selectively monitor monocytes because other cell types, including neutrophils, may be picked up by the agent. 
A No Tx

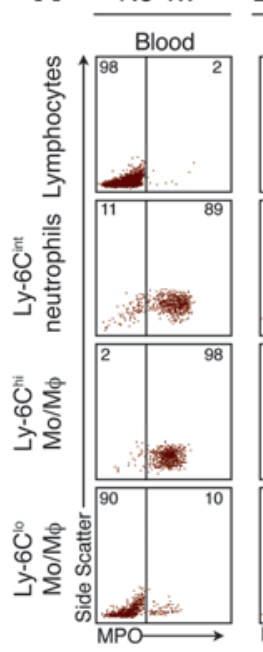

$\underline{\text { Day } 7 \text { after allograft } \mathrm{Tx}}$
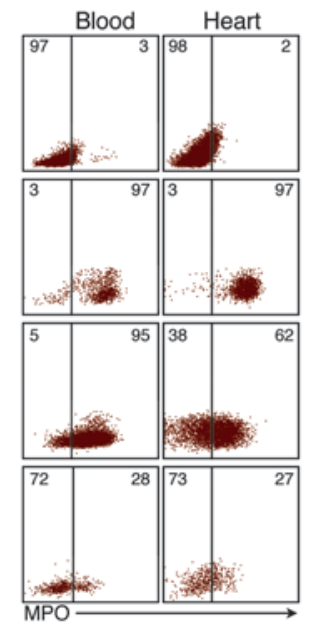

B Isograft day 7
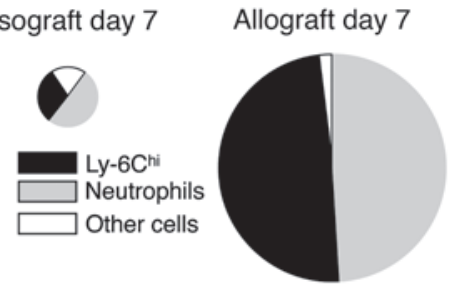

C

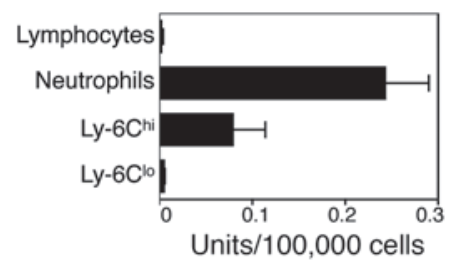

\section{Figure 2}

MPO levels in leukocytes retrieved from blood and transplants. (A) Flow cytometric intracellular MPO staining on lymphocytes, neutrophils, Ly- $6 \mathrm{C}^{\text {hi }}$ monocytes, and Ly-6Clo monocytes. 1 of 3 representative experiments is shown. Numbers indicate percentage of cells in respective gates. (B) Relative contribution of leukocytes to overall MPO expression in allografts and isografts. The area of the pie chart reflects overall MPO content per mg tissue, which is the product of per cell MPO content and the abundance of each cell type in the heart, as determined by flow cytometry. (C) MPO activity $(n=3)$ of lysed sorted leukocytes.
MPO imaging detects rejection in allografts. We then proceeded to image MPO activity in vivo using MRI after injection of MPO-Gd, a targeted gadolinium chelate. The gadolinium-encaging chelator diethylenetriamine penta-acetic acid (DTPA) is derivatized with 2 serotonin moieties that radicalize in MPO-rich tissue, react with each other, and form larger oligomers that exhibit higher relaxivity and slower washout kinetics $(19,20)$. After a set of scout MRI scans, which localized the heterotopic heart graft in the abdominal cavity (Figure 3, A and B), we acquired T1-weighted short axis gradient echo images (21) prior to and up to 2 hours after injection of the agent into allografts in $\mathrm{MPO}^{+/+}$and $\mathrm{MPO}^{-/-}$recipients (Figure 3, C-E). Allografts in wildtype mice showed areas with persistent enhancement, whereas there was no enhancement in MPO-deficient mice.

Continuous imaging allowed us to follow the contrast-to-noise ratio (CNR) in myocardial tissue over time (Figure 4, A and B). In isograft controls, the CNR peaked shortly after injection but rapidly returned to preinjection values, whereas there was consistently increased signal in allografts. Figure 4, C-E, shows MPO signal in representative images of iso- and allografts 2 hours after injection. Signal enhancement in MRI colocalized with immunoreactive staining of MPO (Figure 4F). Enhanced areas were of variable size, shape, and distribution and often localized to the left ventricular free wall (Figure 4G). Notably, many of these areas of rejection would be inaccessible for biopsies in the clinic, which often use a transvenous approach to retrieve tissue for histological analysis from the right ventricle and interventricular septum. For example, strong parenchymal rejection visualized by MPO-targeted MRI in 2 cases shown in Figure 4G mapped only to the left ventricle, a site unreachable with a bioptome.

MPO imaging allows for therapeutic monitoring. Noninvasive diagnostic imaging should be sufficiently sensitive for tracking therapeutic efficacy. We therefore investigated the impact of clinically relevant immunosuppressive therapy (1) on imaging and correlated it with

\section{Figure 3}

MRI of MPO activity. (A and B) Orientation of the heterotopic graft and localizer MRI. (C) Change of CNR from before to 2 hours after MPOGd injection in wild-type and MPO-deficient mice 7 days after allograft transplantation. Data are shown as mean \pm SEM; $n=4-9$ per group; ${ }^{\star} P=0.02$. ( $\mathbf{D}$ and $\mathbf{E}$ ) Representative short axis views of allografts 2 hours after injection of MPO-Gd.

MPO expression, activity, and myeloid cell infiltration. Therapy with cyclosporin, prednisolone, and azathioprine suppressed MPO protein levels (Figure 5A) and MPO activity (Figure 5B) in graft tissue. Flow cytometric enumeration showed substantial reduction of myeloid cells in response to immunosuppression (Figure 5C). These data were corroborated by quantitative immunoreactive staining for MPO, Ly-6C, the macrophage marker F4/80, and the neutrophil marker NIMP-R14 (Figure 5, D and E). MPO-targeted in vivo MRI detected these therapeutic effects. We calculated the change in CNR on images taken before and 2 hours after injection of MPO-Gd. Allograft recipients subjected to immunosuppressive therapy showed no myocardial enhancement and had signal dynamics similar to those of isograft controls (Figure 5, F-G).
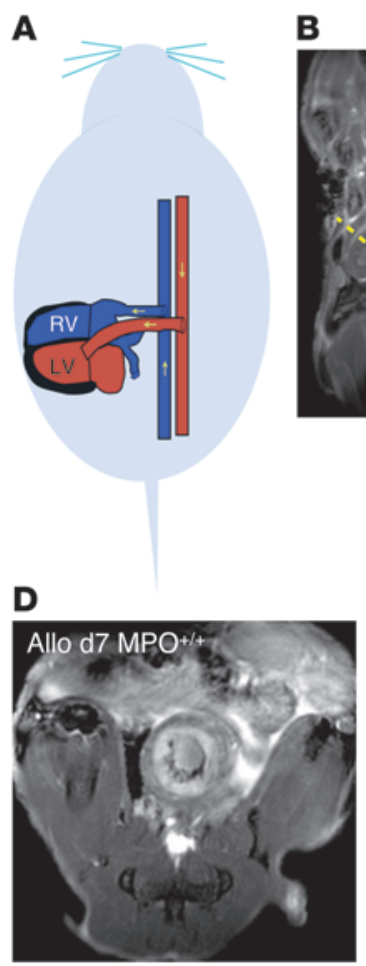

B

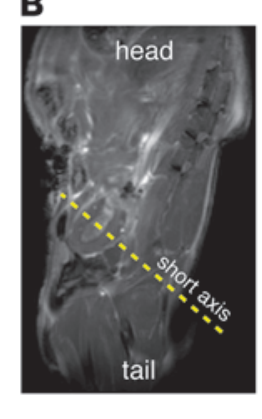

C

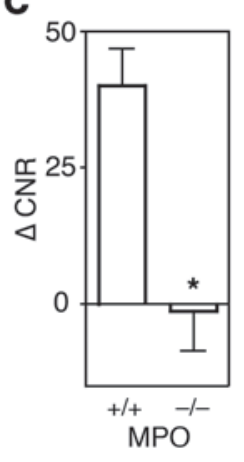

E

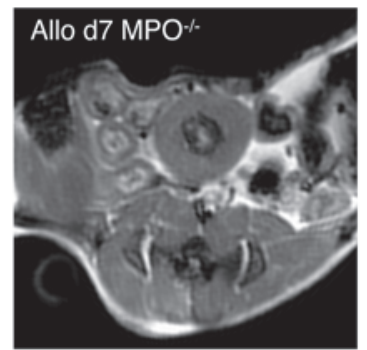



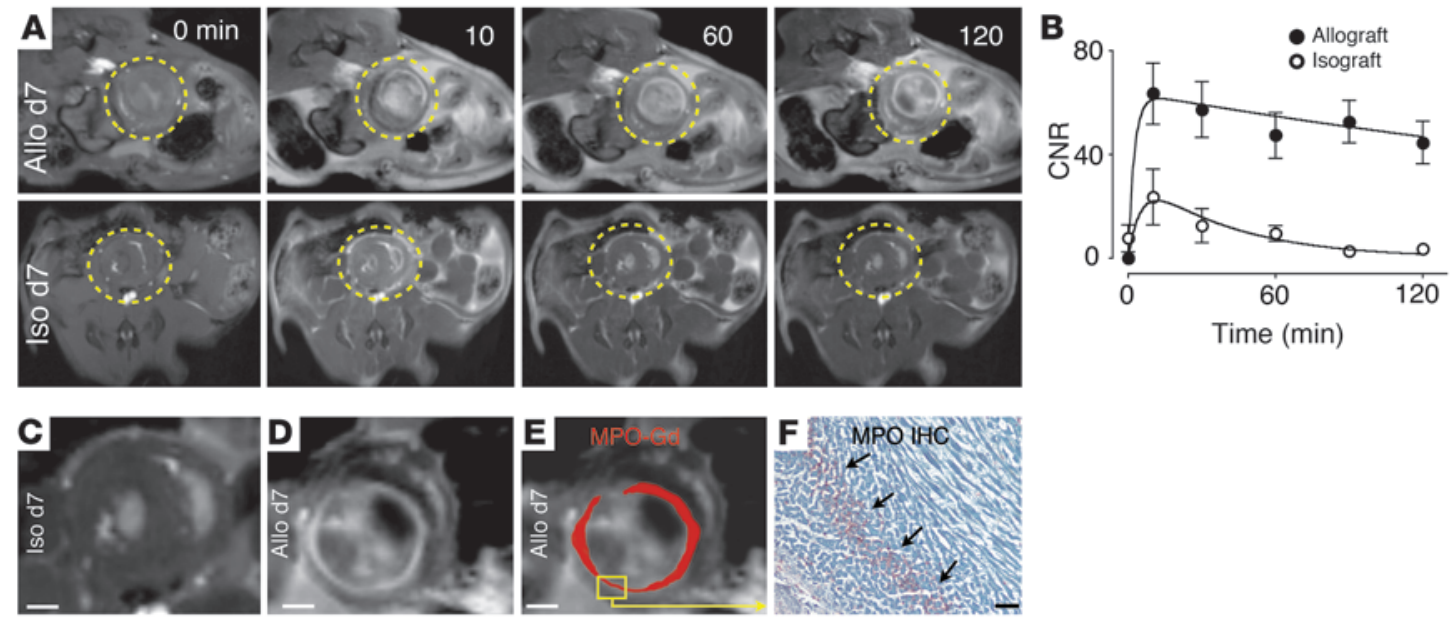

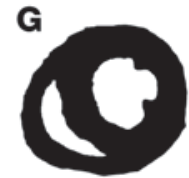

Isograft

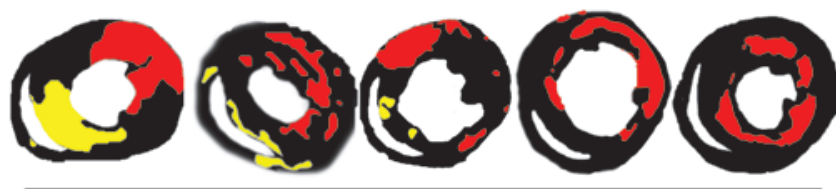

Allograft

\section{Figure 4}

Kinetics and distribution of enhancement. (A and B) T1-weighted short axis MRI of allo- and isografts 7 days after transplantation before and up to 120 minutes after injection of MPO-Gd. Circles indicate location of heart grafts. Data are shown as mean \pm SEM; $n=6$ per group. (C) Magnified MR image of isograft 120 minutes after injection of MPO-Gd. Some focal bright signal reflects spin refreshment effects due to blood flow. Scale bars: $1 \mathrm{~mm}$. (D) Allograft 120 minutes after injection of MPO-Gd. (E) Same data as in D, but the MPO-Gd signal was thresholded and pseudocolored in red. (F) Immunoreactive staining for MPO in the area that enhances in D and E. Scale bar: $20 \mu \mathrm{m}$. (G) Thresholded data of signal enhancement 120 minutes after injection of MPO-Gd show typical enhancement patterns. To highlight the more comprehensive sampling of imaging over the clinical standard, areas that would be accessible to heart biopsies, which are routinely taken from the right ventricular cavity, are color-coded in yellow. Red encodes foci of rejection that could not be reached with routine transvenous right ventricular biopsies.

Dynamic range and sensitivity of MPO imaging are sufficient to detect subtle rejection. A clinically useful method should identify patients who receive suboptimal immunosuppression and detect low-grade rejection. Thus, we thought to test the sensitivity of MPO imaging in cases in which the immune system has been partially suppressed or where the graft is only partially mismatched. First, we imaged cohorts of mice 7 days after allograft transplantation that were treated with daily doses of 10 or $20 \mathrm{mg} / \mathrm{kg}$ prednisolone, respectively. Reflecting low-grade graft rejection, we found intermediate enhancement, i.e., the CNR was higher than in isografts, but lower than in untreated allografts (Figure 6, A and B). Interestingly, the increase of CNR corresponded to the prednisolone dose (Figure 6, A and B). Second, we followed signal dynamics serially in single MHC class II-mismatched grafts $(\mathrm{Bm} 12 \rightarrow \mathrm{B} 6)$. Imaging was done 1,3 , and 5 weeks after transplantation. Here, enhancement increased gradually over time and corresponded to tissue damage and infiltration by myeloid cells as identified by histology (Figure 6, A and C).

Human specimen. We determined the translational potential of MPO-targeted MRI for monitoring rejection of heart transplants. First, we measured MPO activity in human monocyte subsets isolated from blood and found high MPO activity in inflammatory CD $16^{\text {lo }} \mathrm{CD} 14^{+}$monocytes but not in CD $16^{\text {hi }} \mathrm{CD} 14^{\text {lo/neg }}$ monocytes (Figure 7A), findings that were consistent with those observed in mice. We also investigated biopsies from patients in which the International Society of Heart and Lung Transplantation (ISHLT) grade ranged from 0 to $3 \mathrm{R}$ (histology grading; no signs of rejection = grade 0 , severe rejection $=$ grade $3 \mathrm{R})(22)$. Immunohistochemistry revealed increased numbers of $\mathrm{MPO}^{+}$cells in patients with high rejection grades (Figure 7, B and C), indicating that sensing MPO noninvasively in patients will report on rejection.

\section{Discussion}

Myeloid cells contribute considerably to the recruited leukocyte population in acutely rejected organs. Here we show that Ly- $6 \mathrm{C}^{\text {hi }}$ monocytes dominate this cell infiltrate. We identified MPO as an imaging target abundant in Ly-6Chi monocytes and in neutrophils and found that MPO-targeted MRI reliably detected subtle as well as florid organ rejection. Furthermore, MPO imaging reported on the effects of immunosuppressive therapy, and in vivo imaging findings were corroborated with ex vivo gold standard MPO assays.

We used an activatable Gd-chelate, previously shown to be specific for MPO (20), to image acute cardiac rejection. In rejecting hearts, we found strong enhancement 2 hours after injection, a time point when nonactivated MPO-Gd was washed out of healthy myocardium. The enhancement pattern in rejected organs was patchy and in some severe cases formed a circle in the midmyocardium of the left ventricular wall. The existence of these enhancements, together with quantitative data such as CNRs of graft tissue, may be used to identify patients at risk for rejection and to adapt immunosuppressive therapy accordingly. Rapid follow-up imaging sessions could replace repetitive biopsies that are currently necessary to determine the effectiveness of immunosup- 
A

$\begin{aligned} \text { MPO } & \text { Allo d7 } \\ \text { GAPDH } & =-\infty\end{aligned}$

B

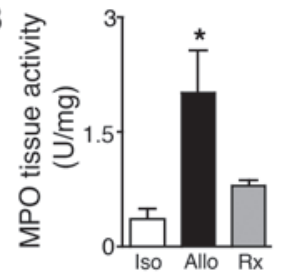

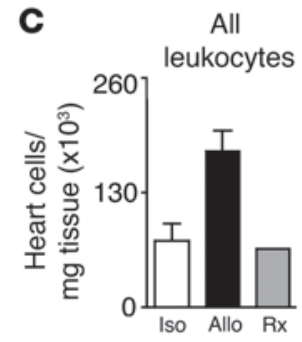
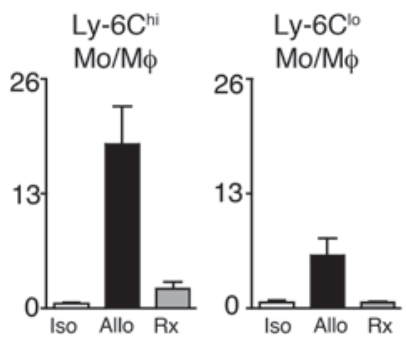

D
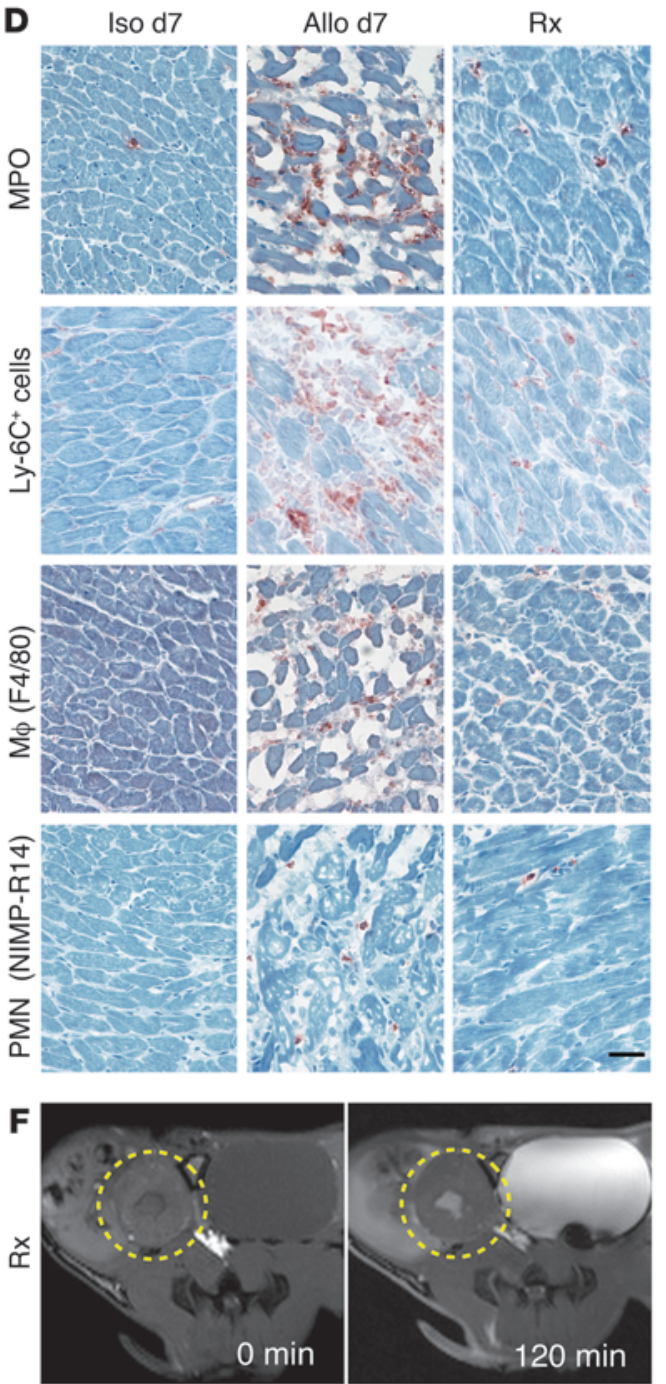

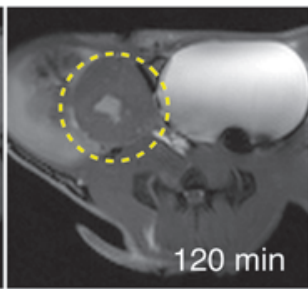

\section{E}
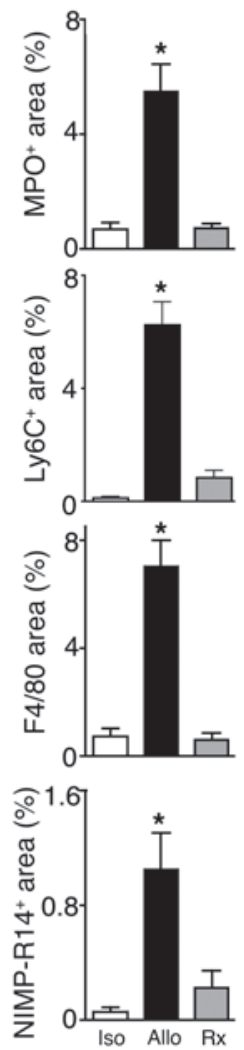

G

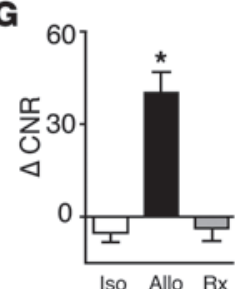

\section{Figure 5}

Effects of clinically relevant immunosuppressive therapy 7 days after transplantation. (A) MPO protein tissue level assessed by Western blotting and (B) biochemical determination of MPO tissue activity in isografts, allografts, and allografts of mice treated with prednisolone, azathioprine, and cyclosporine (Rx). (A) Data are shown as mean \pm SEM; $n=3$ per group. ${ }^{*} P<0.01$ (C) Flow cytometric profiling of leukocytes in the same groups as above. (D and E) Quantitative immunoreactive staining for MPO, Ly-6C, macrophage (F4/80), and neutrophil marker (NIMP-R14) in the 3 groups. $n=3$ mice per group, ${ }^{*} P<0.01$. Scale bar: $10 \mu \mathrm{m}$. (F) Representative MPOGd MRI before and 120 minutes after injection. Circles indicate graft. Next to the graft, the bladder showed enhancement, indicating renal excretion of MPO-Gd. No enhancement was seen in the allograft treated with immunosuppressive therapy. (G) Change of CNR before injection versus 2 hours after injection in iso- and allografts of mice with and without treatment. $n=6$ per group, ${ }^{\star} P<0.05$.

imaging approach for detecting rejection: since the entire organ volume can be assayed in 3 dimensions with high resolution, the approach is more comprehensive and operator independent. Moreover, noninvasive imaging spares the patient complications and discomfort and is devoid of sampling error. The ability to quantify imaging data allows for comparison of serial data sets in 1 individual and between cohorts. The results of the longitudinal trial in single MHC class II-mismatched grafts (Figure 6) suggest that rapid follow-up imaging may be feasible in the clinical arena when effects of adjusted immunosuppressive therapy should be monitored.

Clinical feasibility of imaging organ rejection has been demonstrated previously by targeting phosphatidylserine with a technetium-99m-labeled annexin $V$ probe with specificity for apoptosis (23). Experimentally, approaches to imaging transplant rejection also used myosin-targeted nuclear tracers (24) or magnetic resonance-sensitive iron oxide nanoparticles (25). Of the 2 modalities, MRI is particularly well suited for repetitive imaging of transplanted hearts because it combines high spatial resolution with the ability to measure heart function (26) while avoiding radiation exposure. Iron oxide nanoparticles exploit the phagocytic capacity of myeloid cells to report on organ rejection $(16,25,27)$ and are attractive because they convey high sensitivity. The approach described here is distinct from nanoparticle MRI because the MPO-activatable probe selectively targets a function carried by a subset of destructive leukocytes rather than a function common to all phagocytes. This is especially important given that monocyte subsets are equally labeled by nanoparticles but have contradistinctive roles in inflammation and repair of the damaged myocardium (8). Specifically, MPO-rich Ly- $6 \mathrm{C}^{\text {hi }}$ monocytes promote inflammation and tissue destruction through the release of proteases and inflammatory cytokines such as TNF- $\alpha$ in various inflammatory settings including infection and injury (8), although it is pressive therapy. Many of the inflammatory foci we observed in the mouse would be unreachable with catheter-based biopsies in patients, perhaps explaining the limited sensitivity of heart biopsies. The signal distribution also highlights the advantages of an also possible Ly-6Chi cells play a suppressive role in other conditions such as cancer (28). Second, because the approach described in this work relies on T1-shortening effects of a gadolinium chelate, the technique yields a positive rather than the negative signal 


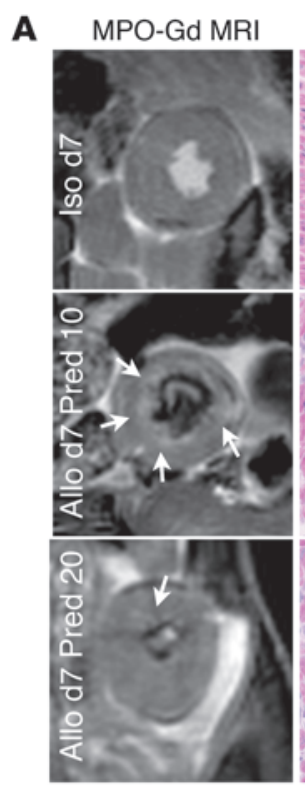

B

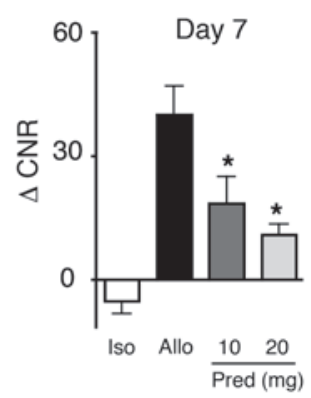

$H \& E$

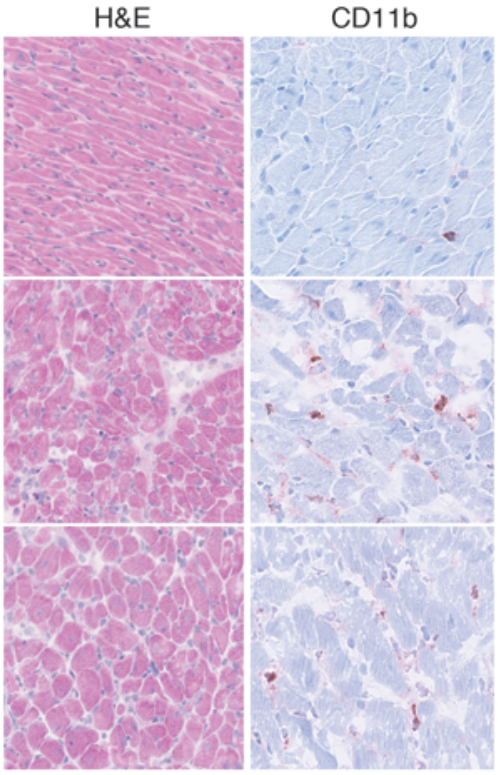

C
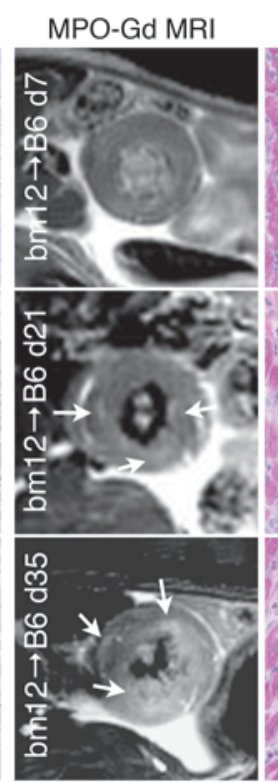

$H \& E$

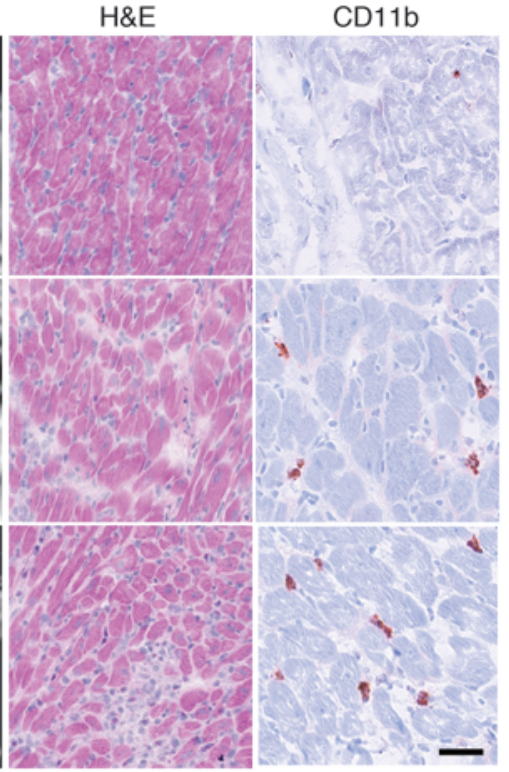

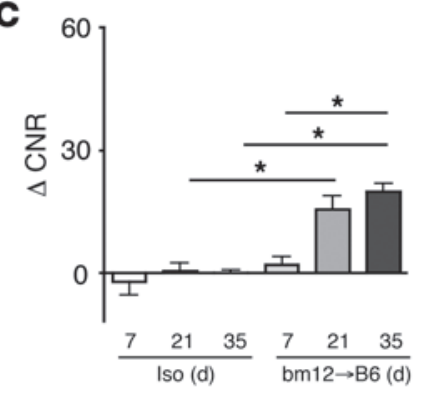

Figure 6

MPO-targeted MRI detects subtle transplant rejection. (A) MR images acquired 2 hours after MPO-Gd in various groups and time points, H\&E histology, and CD11b immunoreactive staining for myeloid cells and H\&E histology. Scale bar: $25 \mu \mathrm{m}$. Arrows indicate enhancing regions after injection of MPO-Gd. (B) MR data acquired in mice treated with insufficient immunosuppression on day 7 after allograft. Pred, prednisolone. $n=4-6$ per group. ${ }^{*} P<0.05$ versus iso- and untreated allograft. (C) Serial imaging in isograft controls $(n=4)$ and single MHC class II mismatch $\left(\mathrm{Bm} 12 \rightarrow \mathrm{B} 6, n=7,{ }^{\star} P<0.05\right)$.

change seen with iron oxide nanoparticles. Signal decay in MRI can also be induced by motion, blood flow, and susceptibility artifacts created by tissue-air interfaces. All of these are common in the heart. Thus, a method that relies on signal increase may confer more specificity. The dynamic range and sensitivity of MPO-Gd MRI shows promise, since we observed enhancement in models of subtle rejection, e.g., in allografted mice on suboptimal immunosuppressive therapy.

Since innate immunity is also involved in ischemia (8), MPOrich cells may invade tissue in diseases other then organ rejection. A patient with a transplanted heart may have progressive coronary artery disease and myocardial ischemia, which would lead to enhancement in MPO-targeted MRI (20). However, the clinical presentation and the pattern of enhancement, which in ischemia should follow coronary anatomy, may help to differentiate rejection from infarction.

In this work, we identified Ly- $6 \mathrm{C}^{\text {hi }}$ monocytes as an abundant, destructive cell population in rejected allografts. The role of myeloid cells in organ rejection is increasingly appreciated, as they closely interact with adaptive immune cells and inflict damage on graft tissue $(17,29,30)$. Thus, monocytes may not only serve as diagnostic biomarkers as shown in our study, but also represent a potential therapeutic target in acute parenchymal rejection. Differentially expressed chemokine receptors and their role in recruitment of subsets (12) may offer an opportunity to target inflammatory monocytes selectively.

\section{Methods}

Transplant surgery. Balb/c, C57BL/6, Bm12, and $\mathrm{MPO}^{-/-}$mice 5 to 8 weeks of age were purchased from the Jackson Laboratories. Hearts were transplanted into the peritoneal cavity of recipients by establishment of an end-toside anastomosis of the donor aorta to the recipient aorta and an end-toside anastomosis of the donor pulmonary trunk to the inferior vena cava as described before (16). The total operative time was in the range of 45-50 minutes. If myocardial contractions were not palpated through the abdomen, the procedure was considered a surgical failure and the mouse was excluded from experiments. We investigated total allo-mismatched grafts (donor mouse, Balb/c, recipient $\mathrm{C} 57 \mathrm{BL} / 6, \mathrm{H} 2^{\mathrm{d}}$ into $\mathrm{H} 2^{\mathrm{b}}$ ), single MHC class IImismatched grafts (donor mouse, Bm12, recipient $\mathrm{C} 57 \mathrm{BL} / 6, \mathrm{H} 2^{\mathrm{bm} 12}$ into $\mathrm{H} 2^{\mathrm{b}}$ ) and compared with syngeneic isografts (donor C57/B6, recipient C57BL/6). A group of total allo-mismatched mice was started on a clinical-type immunosuppressive regimen on the day of surgery: cyclosporin A 


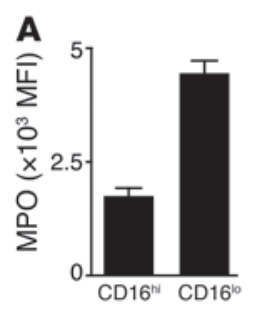

B
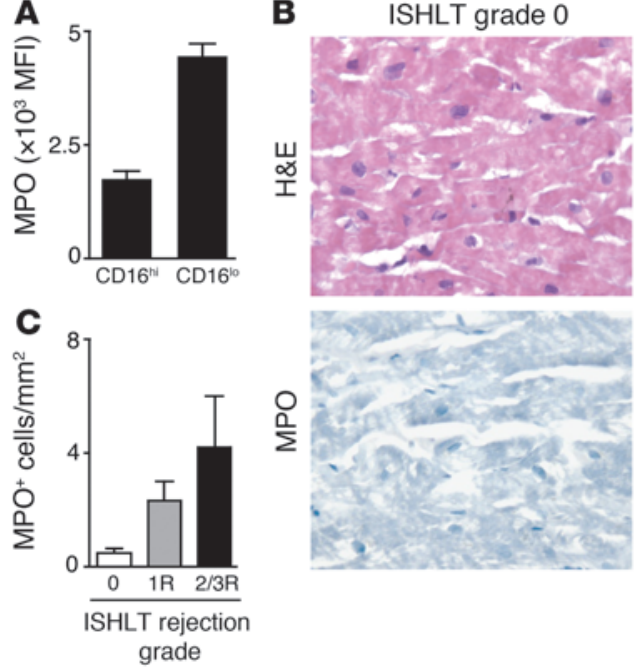

ISHLT grade 3R
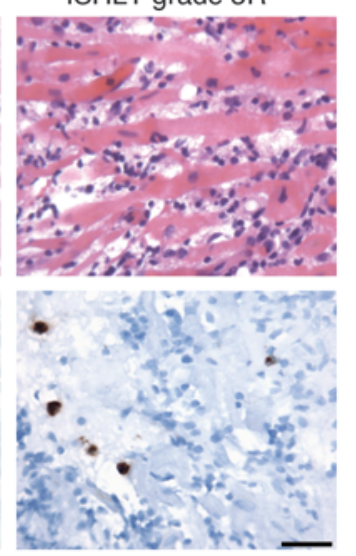

\section{Figure 7}

Translational potential of MPO as an imaging target for organ rejection. (A) MPO protein levels measured by flow cytometry in human blood monocyte subsets. (B) MPO in biopsy samples from patients after heart transplantation. H\&E stain and immunoreactive staining for MPO in representative sections from patient biopsies. Patient on left had no signs of rejection (grade 0 ) and no $\mathrm{MPO}^{+}$cells, whereas patient on the right was graded $3 \mathrm{R}$ (severe rejection/high grade infiltrate) and showed $\mathrm{MPO}^{+}$cells on immunoreactive staining. Scale bar: $20 \mu \mathrm{m}$. (C) Quantification of MPO+ cells in patients with no (ISHLT grade 0), mild (1R), and high grade (2/3R) cellular infiltration. Data are shown as mean \pm SEM.
$(40 \mathrm{mg} / \mathrm{kg} / \mathrm{d}$ s.c.), prednisolone $(10 \mathrm{mg} / \mathrm{kg} / \mathrm{d}$ s.c.), and azathioprine $(10$ $\mathrm{mg} / \mathrm{kg} / \mathrm{d}$ i.p.). Furthermore, we modeled insufficient medical immunosuppressive therapy by treating allografted mice with 10 or $20 \mathrm{mg} / \mathrm{kg} / \mathrm{d}$ prednisolone, respectively. Surgery and imaging were done while mice were anesthetized with isoflurane ( $2 \%$ supplemented with $\mathrm{O}_{2}$ ). The experiments were approved by the Subcommittee on Research Animal Care at Massachusetts General Hospital (Charlestown, Massachusetts, USA).

Flow cytometry. To assess the innate immune cell profile associated with heart transplant rejection, we harvested grafts 3 and 7 days after transplantation, minced the tissue with fine scissors, and placed it into a cocktail of collagenase I, collagenase XI, DNase I, and hyaluronidase (Sigma-Aldrich). The tissue was digested while shaken at $37^{\circ} \mathrm{C}$ for 1 hour and then passed through cell strainers as previously described (8). The following antibodies were used to identify recruited cells and their subsets: anti-CD90-PE, antiB220-PE, anti-CD49b-PE, anti-NK1.1-PE, anti-Ly-6G-PE, anti-Ly-6G-FITC, anti-CD11b-APC, anti-CD11c-biotin, anti-CD11c-FITC, anti-Ly-6C-FITC, anti-Ly-6C-biotin (all from BD Biosciences), anti-F4/80-biotin (AbD Serotec), anti-F4/80-FITC (BioLegend). Strep-PerCP (BD Biosciences) was used to label biotinylated antibodies. Macrophages were identified as $\mathrm{CD} 11 \mathrm{~b}^{\mathrm{hi}}$ (CD90/B220/CD49b/NK1.1/Ly-6G) ${ }^{\text {lo }}$ (F4/80/I-A b CD11c $)^{\text {hi }}$ Ly-6C ${ }^{\text {lo }}$. Monocytes were identified as CD11b $b^{\text {hi }}$ (CD90/B220/CD49b/NK1.1/Ly-6G) ${ }^{\text {lo }}$ $\left(\mathrm{F} 4 / 80 / \mathrm{I}-\mathrm{A}^{\mathrm{b}} / \mathrm{CD} 11 \mathrm{c}\right)^{\mathrm{lo}} \mathrm{Ly}-6 \mathrm{C}^{\mathrm{hi} / \mathrm{lo}}$. Within this population, monocyte subsets were identified as Ly-6Chi or Ly- $6 C^{\text {lo }}$. Neutrophils were identified as CD $11 b^{\text {hi }}$ (CD90/B220/CD49b/NK1.1/Ly-6G) ${ }^{\text {hi }}$ (F4/80/I-A b/CD11c) $)^{\text {lo }}$ Ly-6C+. Cell numbers were calculated as total cells multiplied by percentage of cells within the respective gate. For intracellular staining of MPO (8-F4, Hycult Biotechnology), cells were permeabilized and fixed with a Cytofix/Cytoperm Kit (BD Biosciences). Human monocytes were isolated from peripheral venous blood from healthy donors. To obtain leukocyte suspensions, whole blood was diluted 1:1 with Dulbecco's phosphate-buffered saline (DPBS), and $20 \mathrm{ml}$ was overlaid on a $15-\mathrm{ml}$ density gradient (Ficoll-Paque Plus, density $1.077 \mathrm{~g} / \mathrm{ml}$; GE Healthcare) and centrifuged $\left(20\right.$ minutes, $\left.18 \mathrm{~g}, 18^{\circ} \mathrm{C}\right)$. The mononuclear cell interphase was carefully isolated and washed 3 times with DPBS. Cells were labeled with CD14-PE and CD16 PE-Cy7 (BD Bioscience), and human monocyte subsets were identified as $\mathrm{CD} 16^{\mathrm{lo}} \mathrm{CD} 14^{+}$and $\mathrm{CD} 16^{\mathrm{hi}} \mathrm{CD} 14^{\mathrm{lo} / \mathrm{neg}}$. For intracellular staining of MPO (MPO-FITC; AbD Serotec), cells were permeabilized and fixed with a Cytofix/Cytoperm Kit (BD Biosciences). Sorting was done on a FACSAria (BD Biosciences).

Biochemical assessment of $M P O$ activity. Oxidation of 3,5,3', $5^{\prime}$-tetramethylbenzidine (TMB) by MPO follows the activation of the enzyme by hydro- gen peroxide generating hydroxyl radicals, which then converts TMB to a product that can be monitored at $655 \mathrm{~nm}$. Tissue from allo- and isograft samples was homogenized (Omni International) for 30 seconds on ice in potassium phosphate buffer, $\mathrm{pH}$ 7.0, with acetyltrimethylammonium bromide. Samples were then sonicated, freeze-thawed 3 times, and centrifuged to remove debris. Dilutions of heart samples were assayed by TMB and hydrogen peroxide mixtures (T4444; Sigma-Aldrich) measured with a Cary 50 spectrophotometer (Varian). Slopes of the linear progress curves are converted into molar units with extinction coefficient of $3.9 \times 10^{4}$ $\mathrm{M}^{-1} \times \mathrm{cm}^{-1}$. We performed bicinchoninic acid protein assays (Pierce) to determine total protein concentration of heart samples and to normalize data. Similar assays were done with the total cell lysates of sorted cells, but the data were normalized to 100,000 cells.

Western blot. A tissue portion of grafts was assessed by Western blotting to compare MPO protein content to in vivo MRI data. Samples of graft homogenates were subjected to SDS gel (4\%-15\%; Bio-Rad) electrophoresis. We incubated blots with rabbit primary antibodies for MPO (Millipore), washed with PBS/0.5\% Tween 20, and visualized with Western Lightning (PerkinElmer) oxidation by horseradish peroxidase-conjugated goat anti-rabbit secondary antibody (Jackson ImmunoResearch). To verify that similar amounts of protein were loaded, blots were stripped with Restore solution (Pierce) and the procedure was repeated with anti-GAPDH (Rockland).

Histology. For histological studies, graft tissue was embedded in OCT (Sakura Finetek), and 6- $\mu \mathrm{m}$ thick sections were cut. Sections were stained with antibodies for neutrophils (NIMP R14; Abcam), MPO (NeoMarkers), Ly6C, CD11b (BD Pharmingen), and F4/80 (Abcam). The reaction was visualized as a 3 -step staining procedure using biotinylated secondary antibodies (BA4001; Vector Laboratories) and AEC Substrate Kit (Vector Laboratories). H\&E staining was also performed to study the overall morphology. Slides were viewed with a Nikon Eclipse 50i, and entire slides were digitized automatically at a magnification of $\times 400$ (NanoZoomer 2.0HT; Olympus). Staining was quantified in 5 high-power fields per mouse using semiautomated thresholding in IPLab and expressed as percentage of positive area (Scanalytics).

$\mathrm{H} \& \mathrm{E}$-stained sections of right ventricular endomyocardial biopsies obtained from patients at Massachusetts General Hospital who had undergone orthotopic heart transplantation were evaluated for acute cellular rejection using the revised 2004 ISHLT criteria: 0R, no evidence of acute cellular rejection; $1 \mathrm{R}$, focal lymphocyte predominant infiltrates with 
or without a single associated focus of myocyte injury; $2 \mathrm{R}, 2$ or more foci of lymphocyte predominant infiltrates with associated myocyte injury; $3 \mathrm{R}$, diffuse lymphocyte predominant infiltrates associated with myocyte injury. The endomyocardial biopsies were also stained for MPO by immunohistochemistry using polyclonal primary antibodies (Dako) at 1:700 dilution and prediluted HRP-conjugated secondary antibodies (Ventana) with a Ventana automated staining system. The MPO-positive cells were counted and the values normalized to the myocardial area assessed by morphometric analysis using a Nikon Eclipse E600 microscope equipped with a Hitachi HV-C20 3-CCD digital camera with computer interface and IPLab Spectrum software (Signal Analytics). The studies using human tissue were reviewed and approved by the Partners Human Research Committee (Partners Human Research Office, Boston, Massachusetts, USA).

$M R I$. The MPO-sensitive imaging agent bis-5-hydroxytryptamide-diethylenetriamine-pentaacetate (bis-5HT-DTPA(Gd), MPO-Gd, molecular weight $=866 \mathrm{~g} / \mathrm{mol}$ ) was synthesized by reacting DTPA-bisanhydride with serotonin in dimethylformamide in the presence of an excess of triethylamine, as described previously (19). The agent was injected i.v. at a dose of $0.3 \mathrm{mmol} / \mathrm{kg}$ body weight. Molecular MRI was performed on day 7 after transplantation on a 7 Tesla scanner (Bruker Pharmascan), while mice were anesthetized with isoflurane $\left(1.5 \%, \mathrm{O}_{2} 2 \mathrm{l} / \mathrm{min}\right)$. Single MHC class IImismatched grafts $(\mathrm{Bm} 12 \rightarrow \mathrm{B} 6)$ were imaged serially 1,3 , and 5 weeks after transplantation. After appropriate localizers, we obtained a series of T1-weighted gradient sequences of several short axes of the transplanted heart. The ECG signal of the cardiac graft was isolated from the orthotopic native heart of the recipient by attachment of electrodes to hind limbs.(16) Cine images were obtained with ECG and respiratory gating (SA Instruments) using a gradient echo FLASH sequence and a dedicated mouse cardiac volume coil in bird-cage design (Rapid Biomedical). Imaging param- eters were as follows: echo time (TE), $2.7 \mathrm{~ms} ; 16$ frames per RR interval (TR 7.0-15 ms depending on heart rate); in-plane resolution, $200 \times 200 \mu \mathrm{m}$; slice thickness, $1 \mathrm{~mm}$; NEX 4 . A high flip angle $\left(60^{\circ}\right)$ nulled nonenhancing myocardial tissue (21). Images were analyzed using OsiriX (The Osirix Foundation). Signal intensities were measured by tracing a region of interest that comprised the entire myocardium of the midventricular short axis slice and skeletal muscle. CNRs were calculated as follows: $\mathrm{CNR}=($ target signal in graft - skeletal muscle signal)/(SD of the noise outside of animal).

Statistics. Results are expressed as mean \pm SEM. Data were compared using the unpaired 2-sided Student's $t$ test. For multiple comparisons, we used ANOVA, followed by Bonferroni's post test. $P<0.05$ was considered significant. We used GraphPad Prism 4.0c for Macintosh (GraphPad Software Inc.) for statistical analysis.

\section{Acknowledgments}

The authors thank Cory Siegel, Aleksey Chudnovskiy, and Rostic Gorbatov for technical assistance. This work was supported in parts by NIH grants R01HL095629, U24-CA092782, U01HL080731, K08HL081170, and the EC-Marie Curie grant OIF (Molecular Imaging 39639).

Received for publication January 12, 2010, and accepted in revised form April 28, 2010.

Address correspondence to: Ralph Weissleder or Matthias Nahrendorf, MGH-CSB, CPZN-5206, 185 Cambridge Street, Boston, Massachusetts 02124, USA. Phone: 617.643.0500; Fax: 617.643.6133; E-mail: rweissleder@mgh.harvard.edu (R. Weissleder); mnahrendorf@mgh.harvard.edu (M. Nahrendorf).
1. Miniati DN, Robbins RC. Heart transplantation: a thirty-year perspective. Annu Rev Med. 2002; 53:189-205.

2. Kubo SH, et al. Risk factors for late recurrent rejection after heart transplantation: a multiinstitutional, multivariable analysis. Cardiac Transplant Research Database Group. J Heart Lung Transplant. 1995;14(3):409-418.

3. Gradek WQ, D'Amico C, Smith AL, Vega D, Book WM. Routine surveillance endomyocardial biopsy continues to detect significant rejection late after heart transplantation. J Heart Lung Transplant. 2001;20(5):497-502.

4. Tucker PA 2nd, Jin BS, Gaos CM, Radovancevic B, Frazier OH, Wilansky S. Flail tricuspid leaflet after multiple biopsies following orthotopic heart transplantation: echocardiographic and hemodynamic correlation. J Heart Lung Transplant. 1994; 13(3):466-472.

5. Swirski FK, et al. Ly-6Chi monocytes dominate hypercholesterolemia-associated monocytosis and give rise to macrophages in atheromata. J Clin Invest. 2007;117(1):195-205.

6. Tacke F, et al. Monocyte subsets differentially employ CCR2, CCR5, and CX3CR1 to accumulate within atherosclerotic plaques. J Clin Invest. 2007; 117(1):185-194.

7. Swirski FK, Weissleder R, Pittet MJ. Heterogeneous in vivo behavior of monocyte subsets in atherosclerosis. Arterioscler Thromb Vasc Biol. 2009; 29(10):1424-1432.

8. Nahrendorf $M$, et al. The healing myocardium sequentially mobilizes two monocyte subsets with divergent and complementary functions. J Exp Med. 2007;204(12):3037-3047.

9. Auffray C, et al. CX3CR1+ CD115+ CD135+ common macrophage/DC precursors and the role of CX3CR1 in their response to inflammation. J Exp Med. 2009;206(3):595-606.
10. Serbina NV, Salazar-Mather TP, Biron CA, Kuziel WA, Pamer EG. TNF/iNOS-producing dendritic cells mediate innate immune defense against bacterial infection. Immunity. 2003;19(1):59-70.

11. Geissmann F, Jung S, Littman DR. Blood monocytes consist of two principal subsets with distinct migratory properties. Immunity. 2003;19(1):71-82.

12. Gordon S, Taylor PR. Monocyte and macrophage heterogeneity. Nat Rev Immunol. 2005;5(12):953-964.

13. Ziegler-Heitbrock L. The CD14+ CD16+ blood monocytes: their role in infection and inflammation. J Leukoc Biol. 2007;81(3):584-592.

14. Sunderkotter C, et al. Subpopulations of mouse blood monocytes differ in maturation stage and inflammatory response. J Immunol. 2004;172(7):4410-4417.

15. Hancock WW, Thomson NM, Atkins RC. Composition of interstitial cellular infiltrate identified by monoclonal antibodies in renal biopsies of rejecting human renal allografts. Transplantation. 1983; 35(5):458-463.

16. Christen T, et al. Molecular imaging of innate immune cell function in transplant rejection. Circulation. 2009;119(14):1925-1932.

17. Girlanda R, et al. Monocyte infiltration and kidney allograft dysfunction during acute rejection. $A m J$ Transplant. 2008;8(3):600-607.

18. Swirski FK, et al. Identification of splenic reservoir monocytes and their deployment to inflammatory sites. Science. 2009;325(5940):612-616.

19. Chen JW, Querol Sans M, Bogdanov A Jr, Weissleder R. Imaging of myeloperoxidase in mice by using novel amplifiable paramagnetic substrates. Radiol ogy. 2006;240(2):473-481.

20. Nahrendorf $M$, et al. Activatable magnetic resonance imaging agent reports myeloperoxidase activity in healing infarcts and noninvasively detects the antiinflammatory effects of atorvastatin on ischemia-reperfusion injury. Circulation. 2008;117(9):1153-1160.

21. Yang Z, Berr SS, Gilson WD, Toufektsian MC, French
BA. Simultaneous evaluation of infarct size and cardiac function in intact mice by contrast-enhanced cardiac magnetic resonance imaging reveals contractile dysfunction in noninfarcted regions early after myocardial infarction. Circulation. 2004; 109(9):1161-1167.

22 . Stewart S, et al. Revision of the 1990 working formulation for the standardization of nomenclature in the diagnosis of heart rejection. J Heart Lung Transplant. 2005;24(11):1710-1720.

23. Narula J, et al. Annexin-V imaging for noninvasive detection of cardiac allograft rejection. Nat Med. 2001;7(12):1347-1352.

24. Frist $W$, et al. Noninvasive detection of human cardiac transplant rejection with indium-111 antimyosin (Fab) imaging. Circulation. 1987;76(5 pt 2):V81-V85.

25. Kanno S, et al. Macrophage accumulation associated with rat cardiac allograft rejection detected by magnetic resonance imaging with ultrasmall superparamagnetic iron oxide particles. Circulation. 2001; 104(8):934-938.

26. Aherne $\mathrm{T}$, et al. Magnetic resonance imaging of cardiac transplants: the evaluation of rejection of cardiac allografts with and without immunosuppression. Circulation. 1986;74(1):145-156.

27. Wu YL, et al. In situ labeling of immune cells with iron oxide particles: an approach to detect organ rejection by cellular MRI. Proc Natl Acad Sci U S A. 2006; 103(6):1852-1857.

28. Gabrilovich DI, Ngaraj S. Myeloid-derived suppressor cells as regulators of the immune system. Nat Rev Immunol. 2009;9(3):162-174.

29. Jose MD, Ikezumi Y, van Rooijen N, Atkins RC, Chadban SJ. Macrophages act as effectors of tissue damage in acute renal allograft rejection. Transplantation. 2003;76(7):1015-1022.

30. Wyburn KR, Jose MD, Wu H, Atkins RC, Chadban SJ. The role of macrophages in allograft rejection. Transplantation. 2005;80(12):1641-1647. 